\title{
Transient paraproteinemia after allogeneic hematopoietic stem cell transplantation is an underexplored phenomenon associated with graft versus host disease
}

\author{
Corinne C. Widmer ${ }^{1}$, Stefan Balabanov ${ }^{1}$, Urs Schanz ${ }^{1, *}$ and Alexandre P.A. \\ Theocharides ${ }^{1, *}$ \\ ${ }^{1}$ Division of Hematology, University Hospital Zurich and University of Zurich, Zurich, Switzerland \\ *These authors contributed equally to this work \\ Correspondence to: Corinne C.Widmer, email: corinne.widmer@usz.ch
}

Keywords: paraprotein; allo-HSCT; GvHD; myeloma

Received: August 13, $2017 \quad$ Accepted: October 27, $2017 \quad$ Published: November 15, 2017

Copyright: Widmer et al. This is an open-access article distributed under the terms of the Creative Commons Attribution License 3.0 (CC BY 3.0), which permits unrestricted use, distribution, and reproduction in any medium, provided the original author and source are credited.

\section{ABSTRACT}

The clinical and biological relevance of a paraprotein that newly arises after allogeneic hematopoietic stem cell transplantation (allo-HSCT) in non-myeloma patients is unknown. In this study, the incidence, the course, and the clinical impact of paraproteins found after allo-HSCT were investigated in a cohort of 383 non-myeloma patients. Paraproteinemia after allo-HSCT was more frequent $(52 / 383$ patients, $14 \%)$ than the reported incidence of monoclonal gammopathy of unknown significance (MGUS) in age-matched healthy subjects and, in contrast to MGUS, did not correlate with age. In most patients $(32 / 52,62 \%)$, the paraprotein appeared transiently within the first year after allo-HSCT with a median duration of 6.0 months. Post-allo-HSCT paraproteinemia was significantly associated with graft versus host disease (GvHD) and correlated with a survival benefit within the first year, but not after five years following allo-HSCT. Importantly, patients with post-allo-HSCT paraproteinemia did not progress into a plasma cell myeloma as observed for MGUS inferring a distinct pathogenic mechanism. Skewing of lymphocyte subpopulations and alterations in cytokine levels in GvHD may explain the expansion of a specific plasma cell subset in non-myeloma patients undergoing allo-HSCT. Our data suggests that paraproteinemia after allo-HSCT is a reactive phenomenon rather than the consequence of clonal plasma cell transformation.

\section{INTRODUCTION}

Paraproteins are abnormal immunoglobulins detected by immunofixation and protein electrophoresis. A monoclonal gammopathy of unknown significance (MGUS) is characterized by the production of a paraprotein by clonal, genetically aberrant plasma cells [1]. While the detection of a paraprotein per se is abnormal, its clinical relevance depends on the plasma cell mass and end organ damage. In healthy subjects, the incidence of paraproteinemia increases with age and confers an increased risk for the development of plasma cell myeloma [2]. IgG is the most frequent paraprotein subtype while the incidence of $\operatorname{IgM}$ and $\operatorname{IgA}$ paraproteins is significantly lower, but associated with a higher risk of transformation to plasma cell myeloma [3].

The appearance of paraproteins following allogeneic hematopoietic stem cell (allo-HSCT) and solid organ transplantation has been reported previously [4-9]. However, the clinical and prognostic relevance of post-transplantation paraproteinemia remains unclear. Factors reported to be associated with the development of paraproteinemia following allo-HSCT include an alemtuzumab-based conditioning regimen, reactivation of cytomegalovirus (CMV), and occurrence of chronic graft versus host disease (GvHD) [6,7]. 
In this large retrospective study, we investigated the development of post-allo-HSCT paraproteinemia in 383 non-myeloma patients treated with allo-HSCT and aimed at identifying predictive factors and clinical parameters associated with this phenomenon.

\section{RESULTS}

We analyzed a cohort of 383 non-myeloma patients who underwent allo-HSCT for the development of paraproteinemia after HSCT. Paraproteinemia was detected in $52 / 383(14 \%)$ patients and was equally distributed among age groups $(p=0.723)$ (Figure 1A). Most paraproteins were of IgG subtype (39/52, 75\%) with an equal distribution between kappa and lambda light chain expression (Figure 1B). An IgM paraprotein was found in $5 / 52(9.6 \%)$ patients. In $8 / 52(15.4 \%)$ cases, two paraproteins were detected in the same patient (IgG kappa/IgG lambda, IgM kappa/IgM lambda and IgG/IgM). However, no significant difference in clinical parameters (see below) and survival was observed between patients with one or two paraproteins (data not shown). Almost all paraproteins $(50 / 52,96 \%)$ detected by immunofixation were not quantifiable by protein electrophoresis due to the low level of paraprotein expression, and bone marrow histology did not reveal infiltration by plasma cell myeloma or lymphoma. In the majority of cases (40/52, 77\%), paraproteinemia appeared within the first year after allo-HSCT and the median time to detection of a paraprotein was 6.0 months post-allo-HSCT (range: 0.24-7.05 years, Figure 2). The majority of paraproteins $(32 / 52,62 \%)$ were transient with a median period of detection of 6.0 months (range 2.5-46.8 months). However, in $20 / 52(38 \%)$ patients, the paraproteinemia persisted until death $(n=11)$ or the end of the observation period $(n=9)$. The median observation period of all patients with paraprotein was 37 months (range 5.4-137.1 months). The most frequent cause of death was relapse of the underlying disease (11 patients), while one patient died from GvHD. No evolution to plasma cell myeloma or B-cell lymphoma was observed in patients with post-allo-HSCT paraproteinemia. Accordingly, laboratory parameters associated with the development of plasma cell myeloma did not deteriorate between the first and the last detection of paraproteinemia (Supplementary Table 1 and Supplementary Figure 1).

To identify factors that may predict or be causative for paraprotein development following allo-HSCT we then correlated clinical parameters with the appearance of postallo-HSCT paraproteinemia. The majority of patients in the study were treated for acute leukemia, but no association was identified between the underlying disease and the appearance of paraproteins (260/383, 68\%, Table 1). However, using a Chi-squared test and a log-linear model a significant association was identified between the detection of a paraprotein and a small group of patients with mature T-cell lymphoma, but not with other diseases (Table 1 and Figure 3A).

The median age of patients with paraproteinemia was 45 years (Table 1) and paraproteinemia was not associated with gender $(p=0.299)$. In 12 patients, paraproteinemia occurred after the first year following allo-HSCT (Figure 2), but we did not find significant differences in clinical parameters and survival between patients with early (i.e. <12 months post-allo-HSCT) or late (i.e. $>12$ months post-allo-HSCT) paraproteinemia (data not shown). An increased incidence of postallo-HSCT paraproteinemia has been reported after alemtuzumab-based conditioning [6, 7]. In this study, only five patients obtained alemtuzumab as part of their conditioning regimen, but none of them developed paraproteinemia (Supplementary Table 2). Furthermore, the conditioning regimen and anti-thymocyte globulin (ATG) pretreatment were not associated with the development of paraproteinemia in the binary logistic regression analysis in our study $(p=0.225)$. In addition, neither the hematopoietic stem cell source $(p=0.381)$, nor the type of donor ( $p=0.127)$ or the reactivation of CMV ( $p=0.494)$ had an impact on the appearance of paraproteinemia. Since none of the patients with paraproteinemia developed a clonal plasma cell disorder, we hypothesized that post-allo-HSCT paraproteinemia was the consequence of a perturbed immune system in patients with GvHD. Indeed, the $\chi^{2}$ test for independency and the binary logistic regression both revealed an association between paraproteinemia and GvHD ( $p=0.034$ and $p=0.046$, respectively, Table 1 and Figure 3B) supporting a causative role for GvHD in postallo-HSCT paraproteinemia. Finally, the Kaplan-Meier survival analysis showed a statistically significant survival benefit in the first year after allo-HSCT for patients with paraproteinemia ( $p=0.017, \mathrm{HR}=0.27, \mathrm{CI}=0.08-0.86$ ), which was not observed five years following allo-HSCT $(p=0.13, \mathrm{HR}=0.64, \mathrm{CI}=0.35-1.15)$ (Figure 4).

\section{DISCUSSION}

In this retrospective study, we investigated 383 nonmyeloma patients treated with allo-HSCT and found a high incidence of post-allo-HSCT paraproteinemia compared to the incidence of MGUS in age-matched healthy subjects [3]. However, and in contrast to MGUS, none of the patients with paraproteinemia developed plasma cell myeloma or B-cell lymphoma during the observation period suggesting a distinct pathogenic mechanism. This hypothesis is also supported by the observation that $62 \%$ of post-allo-HSCT paraproteins were only detected transiently in our cohort. In our study $3 / 4$ patients with mature T-cell lymphoma developed paraproteinemia following allo-HSCT, a finding that needs to be validated in a larger cohort of patients. 
Interestingly, we found a significant association between paraprotein development and the occurrence of GvHD as observed previously in a smaller cohort [6]. Disruption of B-cell homeostasis has been implicated in the pathogenesis of GvHD [10-12]. Patients with chronic GvHD (cGvHD) produce excessive levels of B-cell activating factor (BAFF) which promotes B-cell survival and maturation and is associated with the production of pre-germinal center B-cells and post-germinal center plasma-like cells in patients with cGvHD [13]. Moreover, increased levels of BAFF are associated with increased autoantibody production [14]. Therefore, altered B-cell homeostasis following allo-HSCT may lead to aberrant production of immunoglobulins in GvHD as observed in our study. In addition, increased generation of a T-follicular helper cell subset that produces high levels of IL-17 and
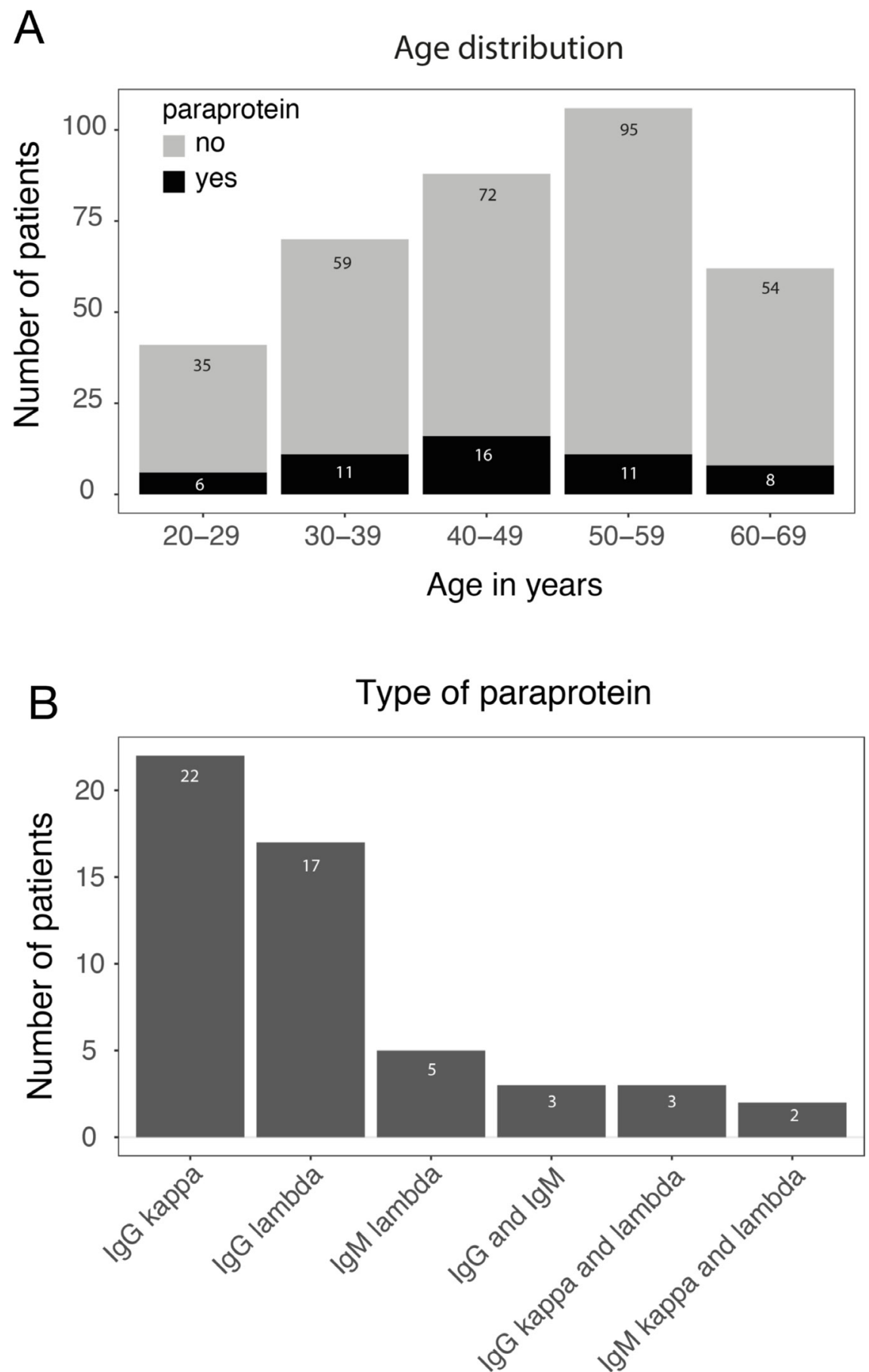

Figure 1: Characterization of post-allo-HSCT paraproteinemia. (A) Number of patients with (black) and without (grey) paraproteins after allo-HSCT stratified according to the age range. (B) Post-allo-HSCT paraprotein subtypes. 
IL-21 and promotes B-cell immunoglobulin secretion may contribute to production of paraproteins [15]. Alterations in T-cell subpopulations may also underlie paraprotein generation in patients receiving allo-HSCT following an alemtuzumab-conditioning as reported previously $[6,7]$. Post-allo-HSCT paraproteinemia was significantly associated with a survival benefit during the first year following allo-HSCT, but not at later time-points. Since most paraproteins appeared transiently during the first year after allo-HSCT and correlated with the occurrence of
GvHD post-allo-HSCT paraproteinemia may hint towards the presence of a graft versus leukemia effect, which could explain the survival benefit observed in the investigated cohort.

In summary, our study investigates the largest nonmyeloma patient cohort to date treated by allo-HSCT for the development of paraproteinemia and suggests an association with GvHD. Importantly, post-allo-HSCT paraproteinemia is mostly transient and does not translate into development of plasma cell myeloma or lymphoma.

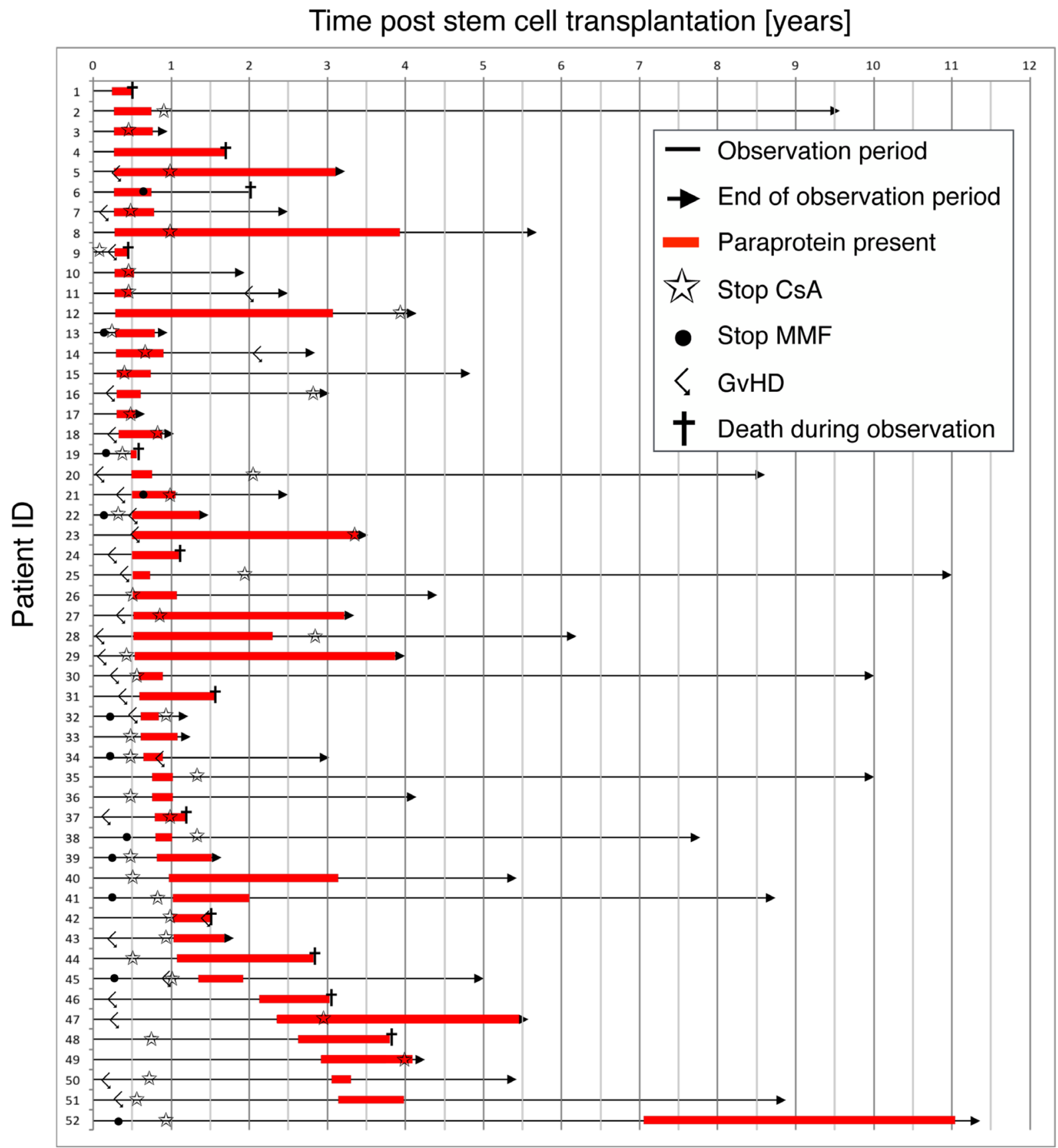

Figure 2: Development of paraproteinemia over time. Each arrow represents the observation period over time of an individual patient with paraproteinemia in the study. The red bar indicates the presence of a paraprotein. Star, Cessation of ciclosporin A; Dot, Cessation of mycophenolate mofetil; Angled arrow, diagnosis of GvHD; Cross, death during observation period. 
Table 1: Characteristics of patients with post-allo-HSCT paraproteinemia

\begin{tabular}{|c|c|c|c|}
\hline Parameter & No paraprotein detected & Paraprotein detected & $p$-value \\
\hline \multicolumn{4}{|l|}{ Age (y) } \\
\hline Median & 48 & 45 & \multirow{2}{*}{0.723} \\
\hline IQR & $36.0-57.5$ & $39.8-53.0$ & \\
\hline \multicolumn{4}{|l|}{ Gender $(n, \%)$} \\
\hline Female & $143(43.2)$ & $27(51.9)$ & \multirow{2}{*}{0.299} \\
\hline Male & $188(56.8)$ & $25(48.1)$ & \\
\hline \multicolumn{4}{|l|}{ Diagnosis $(n, \%)$} \\
\hline ALL & $46(13.9)$ & $9(17.3)$ & \multirow{12}{*}{0.047} \\
\hline AML & $179(54.1)$ & $26(50.0)$ & \\
\hline CLL & $4(1.2)$ & $2(3.8)$ & \\
\hline CML & $24(7.3)$ & $5(9.6)$ & \\
\hline HL & $8(2.4)$ & $2(3.8)$ & \\
\hline $\mathrm{MBCN}$ & $20(6.0)$ & $1(1.9)$ & \\
\hline MDS & $18(5.4)$ & $2(3.8)$ & \\
\hline MDS/MPN & $4(1.2)$ & $0(0.0)$ & \\
\hline MPN & $18(5.4)$ & $2(3.8)$ & \\
\hline MTCN & $1(0.3)$ & $3(5.8)$ & \\
\hline PID & $5(1.5)$ & $0(0.0)$ & \\
\hline SAA & $4(1.2)$ & $0(0.0)$ & \\
\hline \multicolumn{4}{|c|}{ Conditioning $(n, \%)$} \\
\hline MAC & $81(24.5)$ & $13(25.0)$ & \multirow{4}{*}{0.225} \\
\hline $\mathrm{MAC}+\mathrm{ATG}$ & $74(22.4)$ & $16(30.8)$ & \\
\hline RIC & $31(9.4)$ & $1(1.9)$ & \\
\hline $\mathrm{RIC}+\mathrm{ATG}$ & $145(43.8)$ & $22(42.3)$ & \\
\hline \multicolumn{4}{|l|}{ Donor $(n, \%)$} \\
\hline MRD & $157(47.4)$ & $21(40.4)$ & \multirow{4}{*}{0.127} \\
\hline MUD & $135(40.8)$ & $29(55.8)$ & \\
\hline Mismatched & $29(8.8)$ & $1(1.9)$ & \\
\hline Haploidentical & $10(3.0)$ & $1(1.9)$ & \\
\hline \multicolumn{4}{|c|}{ Stem cell source $(n, \%)$} \\
\hline Bone marrow & $50(15.1)$ & $11(21.2)$ & \multirow{3}{*}{0.381} \\
\hline Peripheral blood & $276(83.4)$ & $41(78.8)$ & \\
\hline Cord blood & $5(1.5)$ & $0(0.0)$ & \\
\hline \multicolumn{4}{|l|}{$\operatorname{GvHD}(n, \%)$} \\
\hline Acute & 87 (29.9) & $13(25.0)$ & \multirow{4}{*}{0.034} \\
\hline Chronic & $68(23.4)$ & $18(34.6)$ & \\
\hline Both & $40(13.7)$ & $12(23.1)$ & \\
\hline None & $96(33.0)$ & $9(17.3)$ & \\
\hline \multicolumn{4}{|l|}{$\mathrm{CMV}(n, \%)$} \\
\hline Reactivation & $69(23.3)$ & $15(28.8)$ & \multirow{2}{*}{0.494} \\
\hline No reactivation & $227(76.7)$ & $37(71.2)$ & \\
\hline
\end{tabular}


IQR, interquartile range; Diagnoses: ALL, acute lymphoblastic leukemia; AML, acute myeloid leukemia; CLL, chronic lymphocytic leukemia; CML, chronic myeloid leukemia; HL, Hodgkin lymphoma; MBCN, mature B-cell neoplasms; MDS, myelodysplastic syndrome; MDS/MPN, myelodysplastic/myeloproliferative neoplasms; MPN, myeloproliferative neoplasms; MTCN, mature T-cell neoplasms including Sézary syndrome and mycosis fungoides; PID, Primary immunodeficiency; SAA, severe aplastic anemia. Conditioning regimens: MAC, myeloablative conditioning; MAC $+\mathrm{ATG}$, myeloablative conditioning with anti-thymocyte globulin (Fresenius); RIC, reduced intensity conditioning; RIC+ATG, reduced intensity conditioning with anti-thymocyte globulin. Donor: MRD, mached related donor; MUD, matched unrelated donor (10/10); Mismatched, non-HLA-identical donor; Haploidentical, haploidentical family donor; GvHD, graft versus host disease; CMV, cytomegalovirus.

Future studies will determine the role of GvHD-associated paraproteinemia in the prediction of disease-free survival and investigate the involvement of specific lymphocyte subsets in the pathogenesis of this transient phenomenon.

\section{MATERIALS AND METHODS}

\section{Patients}

The study was approved by the local ethics committee (BASEC-Nr. 2016-01139). 383 patients who underwent allo-HSCT in the Division of Hematology at the University Hospital Zurich, Switzerland, between 2004 and 2014 were retrospectively investigated for the development of post-allo-HSCT paraproteinemia. The following patients were excluded from the study; patients with plasma cell myeloma, autoimmune disease, paraproteinemia before allo-HSCT and patients without documented negative immunofixation prior to allo-HSCT. The observation period started with the date of the alloHSCT and ended December 31, 2015 or with the patient's death. The patient characteristics are outlined in Table 1.

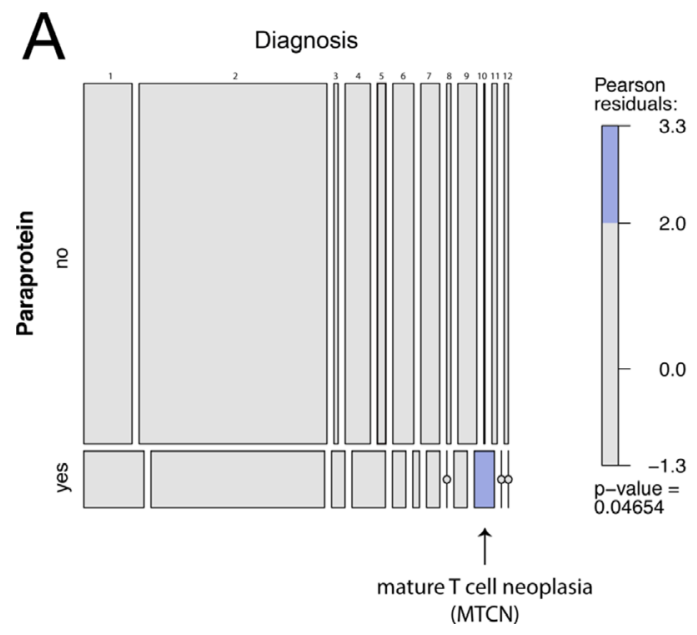

\section{Serum proteins electrophoresis measurement}

Immunofixation and serum protein electrophoresis was performed before and regularly every 3 to 6 months after allo-HSCT. Proteins were separated on an agarosegel followed by immunoprecipitation in situ with monospecific antisera against heavy and light chains (EasyFix Interlab G26, Apteq Switzerland). The gels were interpreted by two independent laboratory technicians. Paraproteins were quantified by densitometry. For the purpose of the study, only monoclonal paraproteins detected by immunofixation were used for correlative studies.

\section{Conditioning regimen and GvHD prophylaxis}

The conditioning regimens used in the study are outlined in Supplementary Table 2. GvHD prophylaxis for all patients consisted of intravenous ciclosporin A (CsA) until engraftment of donor hematopoiesis, followed by oral application with a target whole blood concentration of 150$250 \mu \mathrm{g} / \mathrm{l}$. Patients with myeloablative conditioning (MAC)

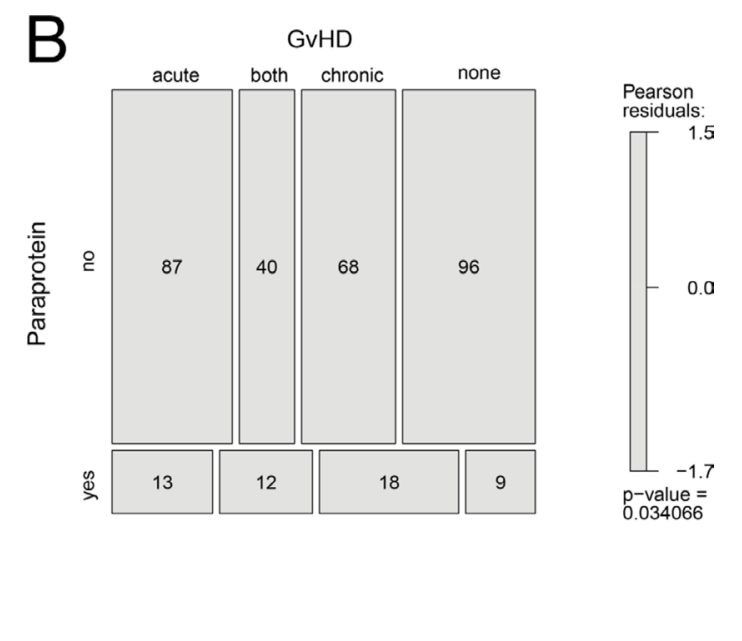

Figure 3: Association of post-allo-HSCT paraproteinemia and clinical parameters depicted in mosaic plots. (A) Association of post-allo-HSCT paraproteinemia and diagnoses according to Table 1. The mosaic plots graphically display data on a loglinear scale. Each horizontal box from left to right represents a diagnosis as presented in Table 1: 1) ALL, acute lymphoblastic leukemia, 2) AML, acute myeloid leukemia, 3) CLL, chronic lymphocytic leukemia, 4) CML, chronic myeloid leukemia, 5) HL, Hodgkin lymphoma, 6) MBCN, mature B-cell neoplasms, 7) MDS, myelodysplastic syndrome, 8) MDS/MPN, myelodysplastic/myeloproliferative neoplasms, 9) MPN, myeloproliferative neoplasms, 10) MTCN, mature T-cell neoplasms including Sézary syndrome and mycosis fungoides, 11) PID, Primary immunodeficiency, 12) SAA, severe aplastic anemia. The blue box indicates a significant association for patients with mature T cell neoplasms (MTCN) and post-allo-HSCT paraproteinemia. The small open dots indicate that no patient was identified in the subgroup. (B) Association of post-allo-HSCT paraproteinemia and GvHD. 


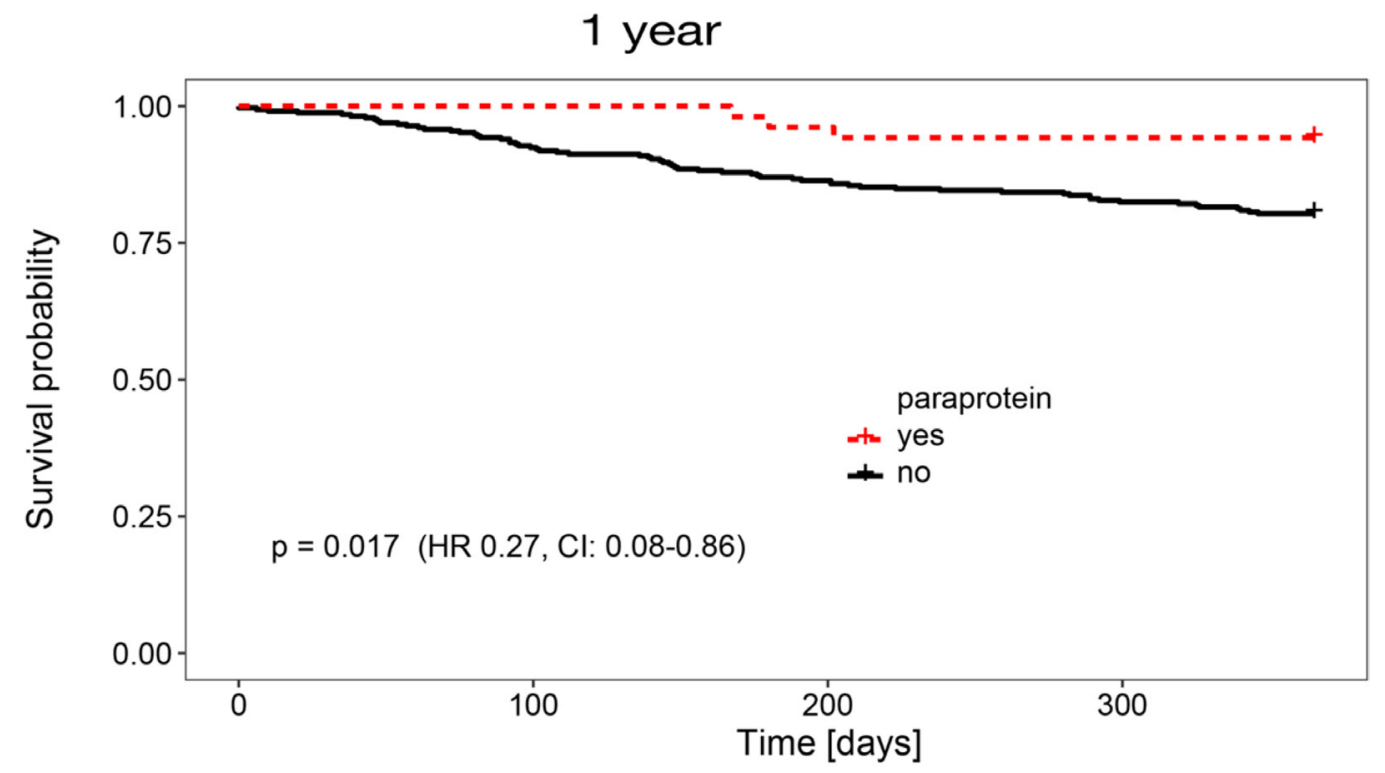

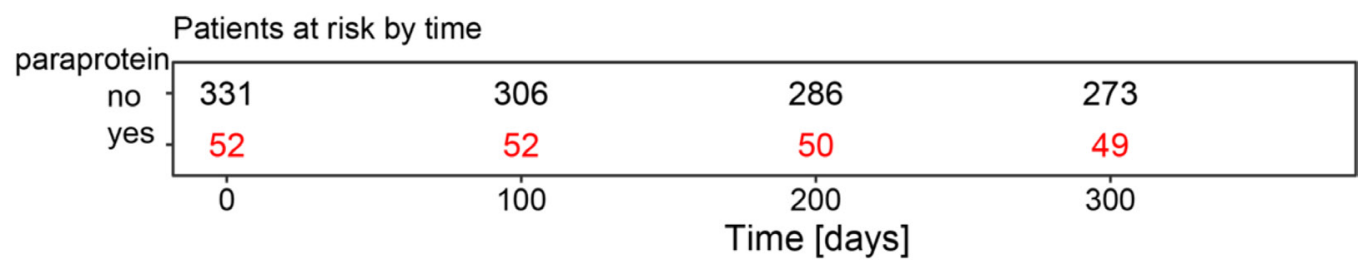

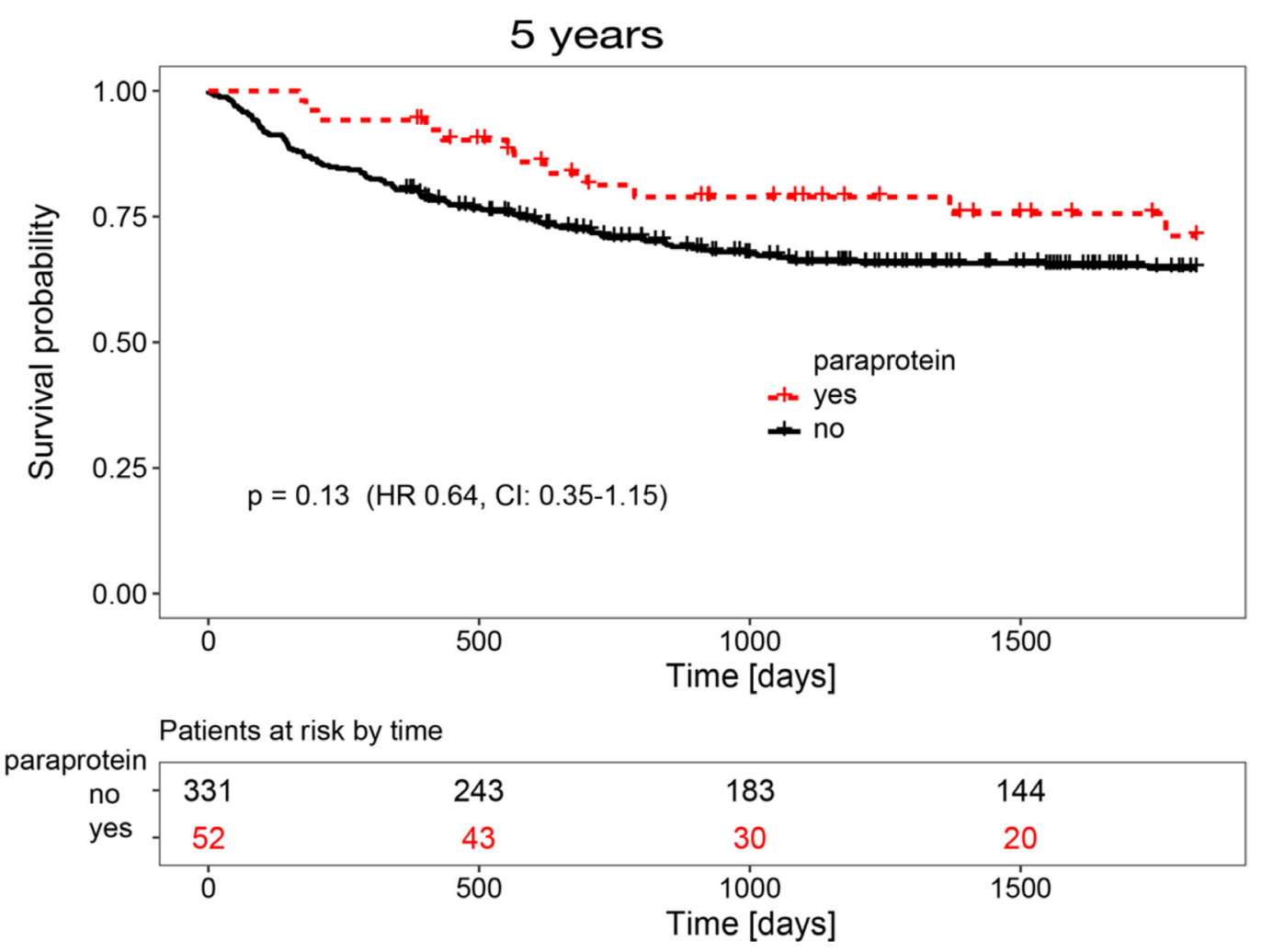

Figure 4: Kaplan-Meier survival analysis for patients with (dashed red line) or without (black line) post-allo-HSCT paraproteinemia. (A) Cumulative survival one year after allo-HSCT. (B) Cumulative survival five years after allo-HSCT. 
received three to four doses of methotrexate (MTX, $15 \mathrm{mg}$ / $\mathrm{m}^{2}$ ) on day 1 and $10 \mathrm{mg} / \mathrm{m}^{2}$ on day 3,6 and 11 after alloHSCT. In addition, mycophenolic mofetil (MMF, $2 \mathrm{~g} / \mathrm{d}$ ) was given to patients with reduced intensity conditioning (RIC) until day 28 in related donor and day 56 in unrelated donor allo-HSCT. In the absence of GvHD immunosuppression was tapered starting at day 100 following allo-HSCT.

\section{Graft versus host disease}

Acute (aGvHD) and chronic GvHD (cGvHD) were defined according to the National Institutes of Health Consensus Development Project criteria [16]. GvHD of the eye and skin was diagnosed clinically. GvHD of the intestine and the liver was documented by histological analysis. Documentation on GvHD development was not available in 40 patients.

\section{Cytomegalovirus reactivation}

CMV copy number quantification was performed twice weekly by polymerase chain reaction for the first 120 days following allo-HSCT. CMV reactivation was defined as a CMV copy number increase above $1000 / \mu 1$. Patients with documented CMV reactivation obtained antiviral treatment. Documentation on CMV reactivation was not available in 35 patients.

\section{Statistical analysis}

All statistical analyses were performed using the statistical software environment R v.3.2.2 (www.r-project. org) with the R packages tableone 0.8.1, compareGroups, ved, survival and survminer. Comparison of categorical data between groups was performed with the Chi-squared test. Furthermore, categorical data for paraprotein, diagnosis and GvHD were analyzed using log-linear modeling and were visualized by mosaic plots. For the estimation of survival curves Kaplan-Meier methods were used. Comparison of overall survival between groups of patients with or without paraproteinemia was performed using the log-rank test and Cox's proportional hazard model (Cox regression).

\section{Abbreviations}

ALL: Acute lymphoblastic leukemia; Allo-HSCT: Allogeneic hematopoietic stem cell transplantation; AML: Acute myeloid leukemia; ATG: Anti-thymocyte globulin; CLL: Chronic lymphocytic leukemia; CML: Chronic myeloid leukemia; CMV: Cytomegalovirus; CsA: Ciclosporin A; GvHD: Graft versus host disease; HL: Hodgkin lymphoma; IQR: Interquartile range; MAC: Myeloablative conditioning; MBCN: Mature B-cell neoplasms; MDS: Myelodysplastic syndrome; MDS/ MPN: Myelodysplastic/myeloproliferative neoplasms; MGUS: Monoclonal gammopathy of unknown significance; MMF: Mycophenolic mofetil; MPN: Myeloproliferative neoplasms; MRD: Mached related donor; MTCN: Mature T-cell neoplasms including Sézary syndrome and mycosis fungoides; MTX: Methotrexate; MUD: matched unrelated donor (10/10); PID: Primary immunodeficiency; RIC: Reduced intensity conditioning; SAA: Severe aplastic anemia.

\section{Author contributions}

CW collected data, performed data analysis, and wrote the manuscript. SB performed data analysis. US supervised the study. AT collected data, wrote the manuscript and supervised the study.

\section{ACKNOWLEDGMENTS}

The authors would like to thank Prof. Markus G. Manz for intellectual input and advice.

\section{CONFLICTS OF INTEREST}

None.

\section{FUNDING}

AT is a recipient of a Cloëtta medical research position.

\section{REFERENCES}

1. Bolli N, Avet-Loiseau H, Wedge DC, Van Loo P, Alexandrov LB, Martincorena I, Dawson KJ, Iorio F, NikZainal S, Bignell GR, Hinton JW, Li Y, Tubio JM, et al. Heterogeneity of genomic evolution and mutational profiles in multiple myeloma. Nat Commun. 2014; 5:2997. https:// doi.org/10.1038/ncomms3997.

2. Kyle RA. Monoclonal gammopathy of undetermined significance. Natural history in 241 cases. Am J Med. 1978; 64:814-26. https://doi.org/10.1016/0002-9343(78)90522-3.

3. Aguzzi F, Bergami MR, Gasparro C, Bellotti V, Merlini G. Occurrence of monoclonal components in general practice: clinical implications. Eur J Haematol. 1992; 48:192-95. https://doi.org/10.1111/j.1600-0609.1992.tb01584.x.

4. Gerritsen EJ, van Tol MJ, Lankester AC, van der WeijdenRagas CP, Jol-van der Zijde CM, Oudeman-Gruber NJ, Radl J, Vossen JM. Immunoglobulin levels and monoclonal gammopathies in children after bone marrow transplantation. Blood. 1993; 82:3493-502. http://www. bloodjournal.org/content/82/11/3493.

5. Nagashima T, Muroi K, Kawano-Yamamoto C, Komatsu N, Ozawa K. Paraproteinemia after hematopoietic stem cell transplantation. Leuk Lymphoma. 2004; 45:135-37. https:// doi.org/10.1080/1042819031000139729. 
6. Lim ZY, Ingram W, Brand R, Akthari M, Milojkovic D, Ho AY, Devereux S, Pagliuca A, Duarte RF, Mufti GJ. Clonal gammopathies following alemtuzumab-based reduced intensity conditioning haematopoietic stem cell transplantation: association with chronic graft-versushost disease and improved overall survival. Bone Marrow Transplant. 2007; 40:747-52. https://doi.org/10.1038/ sj.bmt. 1705805 .

7. Medd P, Littlewood S, Danby R, Malladi R, Clifford R, Wareham D, Jeffery K, Ferry B, Roberts D, Peniket A, Littlewood T. Paraproteinaemia after allo-SCT, association with alemtuzumab-based conditioning and CMV reactivation. Bone Marrow Transplant. 2011; 46:993-99. https://doi.org/10.1038/bmt.2010.244.

8. Pageaux GP, Bonnardet A, Picot MC, Perrigault PF, Coste V, Navarro F, Fabre JM, Domergue J, Descomps B, Blanc P, Michel H, Larrey D. Prevalence of monoclonal immunoglobulins after liver transplantation: relationship with posttransplant lymphoproliferative disorders. Transplantation. 1998; 65:397-400. https://doi. org/10.1097/00007890-199802150-00018.

9. Lemoine A, Pham P, Azoulay D, Saliba F, Emile JF, Saffroy R, Broet P, Bismuth H, Samuel D, Debuire B. Detection of gammopathy by serum protein electrophoresis for predicting and managing therapy of lymphoproliferative disorder in 911 recipients of liver transplants. Blood. 2001; 98:1332-38. https://doi.org/10.1182/blood.V98.5.1332.

10. Kelsey SM, Lowdell MW, Newland AC. IgG subclass levels and immune reconstitution after $\mathrm{T}$ cell-depleted allogeneic bone marrow transplantation. Clin Exp Immunol. 1990; 80:409-12. https://doi.org/10.1111/j.1365-2249.1990.tb03302.x.

11. Corre E, Carmagnat M, Busson M, de Latour RP, Robin M, Ribaud P, Toubert A, Rabian C, Socié G. Longterm immune deficiency after allogeneic stem cell transplantation: b-cell deficiency is associated with late infections. Haematologica. 2010; 95:1025-29. https://doi. org/10.3324/haematol.2009.018853.

12. Allen JL, Fore MS, Wooten J, Roehrs PA, Bhuiya NS, Hoffert T, Sharf A, Deal AM, Armistead P, Coghill J, Gabriel DA, Irons R, Essenmacher A, et al. B cells from patients with chronic GVHD are activated and primed for survival via BAFF-mediated pathways. Blood. 2012; 120:2529-36. https://doi.org/10.1182/blood-2012-06-438911.

13. Sarantopoulos S, Stevenson KE, Kim HT, Cutler CS, Bhuiya NS, Schowalter M, Ho VT, Alyea EP, Koreth J, Blazar BR, Soiffer RJ, Antin JH, Ritz J. Altered B-cell homeostasis and excess BAFF in human chronic graftversus-host disease. Blood. 2009; 113:3865-74. https://doi. org/10.1182/blood-2008-09-177840.

14. Sarantopoulos S, Stevenson KE, Kim HT, Bhuiya NS, Cutler CS, Soiffer RJ, Antin JH, Ritz J. High levels of $\mathrm{B}$-cell activating factor in patients with active chronic graftversus-host disease. Clin Cancer Res. 2007; 13:6107-14. https://doi.org/10.1158/1078-0432.CCR-07-1290.

15. Forcade E, Kim HT, Cutler C, Wang K, Alho AC, Nikiforow S, Ho VT, Koreth J, Armand P, Alyea EP, Blazar BR, Soiffer RJ, Antin JH, Ritz J. Circulating T follicular helper cells with increased function during chronic graft-versus-host disease. Blood. 2016; 127:2489-97. https://doi.org/10.1182/ blood-2015-12-688895.

16. Jagasia MH, Greinix HT, Arora M, Williams KM, Wolff D, Cowen EW, Palmer J, Weisdorf D, Treister NS, Cheng GS, Kerr H, Stratton P, Duarte RF, et al. National Institutes of Health Consensus Development Project on Criteria for Clinical Trials in Chronic Graft-versus-Host Disease: I. The 2014 Diagnosis and Staging Working Group report. Biol Blood Marrow Transplant. 2015; 21:389-401.e1. https:// doi.org/10.1016/j.bbmt.2014.12.001. 